Voix et Images

voixetimages

\title{
Constellation classique
}

Lucie Robert

Volume 14, numéro 2 (41), hiver 1989

L’édition littéraire au Québec

URI : https://id.erudit.org/iderudit/200785ar

DOI : https://doi.org/10.7202/200785ar

Aller au sommaire du numéro

\section{Éditeur(s)}

Université du Québec à Montréal

\section{ISSN}

0318-9201 (imprimé)

1705-933X (numérique)

Découvrir la revue

Citer cet article

Robert, L. (1989). Constellation classique. Voix et Images, 14(2), 353-356.

https://doi.org/10.7202/200785ar d'utilisation que vous pouvez consulter en ligne.

https://apropos.erudit.org/fr/usagers/politique-dutilisation/ 


\section{Dramaturgie}

\section{Constellation classique}

\section{par Lucie Robert, Université du Québec à Montréal}

Curieux destin que celui de certains textes dramatiques, sans cesse réćcrits, réédités, présentés à la scène dans une nouvelle version. Tel est le cas notamment d'Au cour de la rose ${ }^{1}$ que Pierre Perrault reprend depuis trente ans et qui aura été en fin de compte le seul texte dramatique publié par son auteur si l'on exclut «Les pierres en vrac», œuvre de jeunesse parue en 1949 dans le Manteau d'Arlequin, à l'époque du collège Sainte-Marie. Curieuse idée également que de faire de ce texte le premier de la collection Typo-théâtre aux Éditions de l'Hexagone. Car, en fait, Au cœur de la rose appartient à nos classiques d'avant la révolution tranquille, tant par le sujet qu'elle aborde que par le langage qu'elle utilise.

Une fille est amoureuse d'un marin qui doit repartir. Aussi devra-t-elle se contenter d'épouser le Boiteux et d'envisager un avenir qui ressemble en tous points au passé. La mer, le phare, le marin, représentent pour cette fille la route vers la liberté, vers un autre monde. La terre n'est pas nommée, dans cette pièce, pas plus que les personnages, ce qui interdit tout rapprochement avec un référent qui serait québécois ou national. Il demeure intéressant tout de même de voir comment, dans cette dramaturgie, la Fille renonçe à l'étranger et prend possession de la terre, accomplissant en cela le vœu de ses ancêtres et de ses parents. Présentée d'abord à la télévision de Radio-Canada dans une réalisation de Paul Blouin, en 1958, Au cœur de la rose a été mise en scène par Jean-Guy Sabourin et créée le 7 février 1963 par les Apprentis-Sorciers. La présente édition respecte le texte de cette mise en scène, texte très écrit, si on peut dire, où les personnages parlent la même langue que les princesses et les princes de Charles Perreault. L'intérêt premier de cette nouvelle édition demeure la préface de Madeleine Greffard qui offre de la pièce une nouvelle interprétation. Elle analyse l'affrontement de deux discours: celui du désir et celui de la société qui s'y oppose et remarque en outre que le désir est ici porté par une femme, une femme jeune, aidée par sa mère et que trahira un homme, jeune lui aussi, avec l'appui du père. 


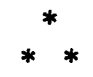

Dans sa dernière pièce, Demande de travail sur les nébuleuses ${ }^{2}$, créée au Théâtre d'aujourd'hui le 2 novembre dernier dans une mise en scène de Yanick Auer, Jovette Marchessault utilise le même langage dépouillé d'identité. Des personnages définis par leur seule fonction, le Père, la Mère, la Fille et le Fils, représentent la Famille nord-américaine, anonyme et stéréotypée à souhait pour les besoins de la démonstration. Projection vers l'avenir, le texte de Marchessault possède tous les défauts des pièces antérieures de l'auteure et aucune de leurs qualités. La division sexuelle du travail et des valeurs, qui sert de moteur à la pièce, fait porter aux femmes et aux jeunes l'espoir d'une vie meilleure, et aux hommes plus âgés l'odieux du présent et le poids des erreurs passées. Aussi les accusations qui tombent sur le Père sont-elles prévisibles dans leur univocité. Marchessault a toutefois le mérite de soulever un ensemble de questions pertinentes quant à l'avenir de la planète. Cette dimension planétaire de l'interrogation entraîne avec elle une série de métaphores galactiques dont témoignent le titre de la pièce et le nom du seul personnage identifié, Stella, correspondante de la Mère. Se pose également le problème du vieillissement: Nous vivons une période difficile où vieillir est condamnable, dit la Mère $[. .$.$] et$ suspect, ajoute le Père. Aussi la pièce baigne-t-elle dans la problématique du temps, que l'on remarque dans les conflits entre les générations, dans la promesse d'une naissance et encore dans cette demande d'emploi, inscrite dans le titre, qui concerne les parents. La réflexion sur l'écriture qui traversait jusqu'à présent la dramaturgie de Jovette Marchessault où nombres d'écrivaines et d'écrivains se trouvaient mis en scène est ici plus ténue et d'autant moins intéressante. Le texte se contente de discussions abstraites sur cet ensemble de problèmes, que la mise en scène, selon les critiques au moment de la création, n'était pas arrivée à rendre vivants. Le texte non plus, faut-il ajouter.

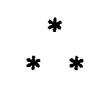

Marie-Antoine, opus $\mathbf{1}^{3}$, de Lise Vaillancourt est la troisième pièce publiće dans la collection Théâtre aux Herbes rouges. Créée à la salle Fred-Barry, le 19 octobre 1984, par le Théâtre expérimental des femmes, dans une mise en scène de Pol Pelletier, le texte, réécrit longuement pour les fins de la publication, est, selon la note liminaire, un grand opéra en mots. Inspiré largement des contes pour enfants, du moins en ce qui concerne la création de personnages et l'atmosphère générale, au point de commencer par Il était une fois, gna, gna, gna, le texte présente le monde du point de vue d'une enfant. L'histoire se passe au début du vingtième siècle dans un château victorien, à Saint-Blaise, et elle prend la forme de mémoires, les mémoires de la fille de la maison, MarieAntoine, qui commence sa première réplique en disant Je prenais des notes dans mon cahier personnel, et qui enchaîne en disant $J^{\prime}$ avais cinq ans et demie et je ne parlais pas encore. Aussi la pièce présente-t-elle une dynamique particulière où les personnages évoluent dans des genres différents, untel vivant 
un mélodrame, un autre étant installé dans un vaudeville, sous le regard incisif d'une enfant muette et plus vraisemblablement autiste. Si le père de MarieAntoine, baron de la Haute-Frénésie, en vient à s'identifier à Tchékhov et à le citer longuement, sa fille finit par ressembler à la Bérénice Einberg de Réjean Ducharme, créant même son propre langage que les adultes n'entendent pas mais qui lui permet de communiquer avec son double, Léa. Une des pièces les plus intéressantes des dernières années, pour la création de personnages et le travail sur le langage, Marie-Antoine, opus 1 demeure un texte dense, un peu trop dense pour la scène, d'autant que les répliques et les tableaux sont ponctués de pièces musicales, qui vont d'un extrait de Carmen jusqu'à Carmina Burana.

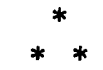

David Lonergan, directeur artistique du Théâtre Pince-farine exerce son métier dans le Bas-du-Fleuve et dans la Gaspésie, loin des centres urbains et de la concurrence artistique qui conduit les auteurs et les metteurs en scène à développer sans cesse la recherche tant sur les formes que sur les thèmes abordés. Publiée par les Éditions coopératives de l'Est, à Rimouski, sa sixième pièce, les Otages ${ }^{4}$, ne reflète pas cette concurrence. Comédie satirique de facture classique, la pièce raconte une histoire, la seule pour tout dire qu'il m'a été donné de lire depuis plusieurs mois, une histoire drôle où les personnages portent des noms et des prénoms rassurants: Emmy et Martine Labrie, Georges et Thérèse Bernatchez, Florence Lagarde. Le seul personnage qui (d)étonne est celui de Louis Darbellay. Normal, il est Suisse, sans travail, immigrant illégal et il vient commettre un hold-up au magasin général des Labrie dont la caisse est vide en raison de l'état général de l'économie gaspésienne et du dernier «emprunt» réalisé par la fille de la maison! Désespéré, Louis, sur les conseils de la propriétaire, fonde un regroupement terroriste et prend en otage toutes les personnes présentes. Celles-ci toutefois approuvent les revendications mises de l'avant par le voleur et adhèrent au regroupement terroriste. Il ne reste donc plus d'otages dans la maison. Comment alors obtenir la rançon? La pièce continue ainsi sans compromis dans la description de la réalité économique de cette région du Québec, avec un sens de l'humour devenu grinçant à la longue, dans une écriture classique destinée à un public peu au fait de recherches formalistes. Le résultat est intéressant et fort réussi, démontrant par le fait même qu'il est encore possible d'écrire des comédies légères de qualité et que le théâtre d'été n'a aucune raison d'être aussi débilitant qu'il l'est devenu depuis quelques années.

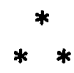

Il faut en outre souligner la parution du dernier numéro des cahiers de théâtre Jeu ${ }^{5}$, numéro thématique intitulé Échos shakespeariens, qui prend la relève des metteurs en scène, pour tenter une réflexion sur les conditions et les difficultés de traduire, de critiquer et de représenter l'œuvre de William Shakespeare sur les scènes du Québec. Y sont évaluées en particulier les 
productions d'Omnibus, le Cycle des rois, du Thêâtre du Nouveau Monde, le Songe d'une nuit d'été, dont la mise en scène était assurée par Robert Lepage, et du Thêâtre expérimental des femmes, l'audacieuse Tempête, mise en scène par Alice Ronfard et où s'est particulièrement illustrée Françoise Faucher dans le rôle de Prospéro. On lira également dans cette livraison l'article de Marcel Fortin sur le théâtre chinois des années 1980.

1 Pierre Perrault, Au cœur de la rose. Pièce en trois actes, troisième version, préface de Madeleine Greffard, Montréal, l'Hexagone, 1988, 159 p. (Typo-théâtre).

2 Jovette Marchessault, Demande de travail sur les nébuleuses, présentation de Pierre Filion, Montréal, Leméac, 1988, 114 p. (Théâtre, n 170).

3 Lise Vaillancourt, Marie-Antoine, opus 1, Montréal, les Herbes rouges, 1988, 97 p.

4 David Lonergan, les Otages, Rimouski, les Éditions coopératives de l'Est du Québec, 1987, 100 p.

5 Jeu, no 48 , septembre 1988,224 p.

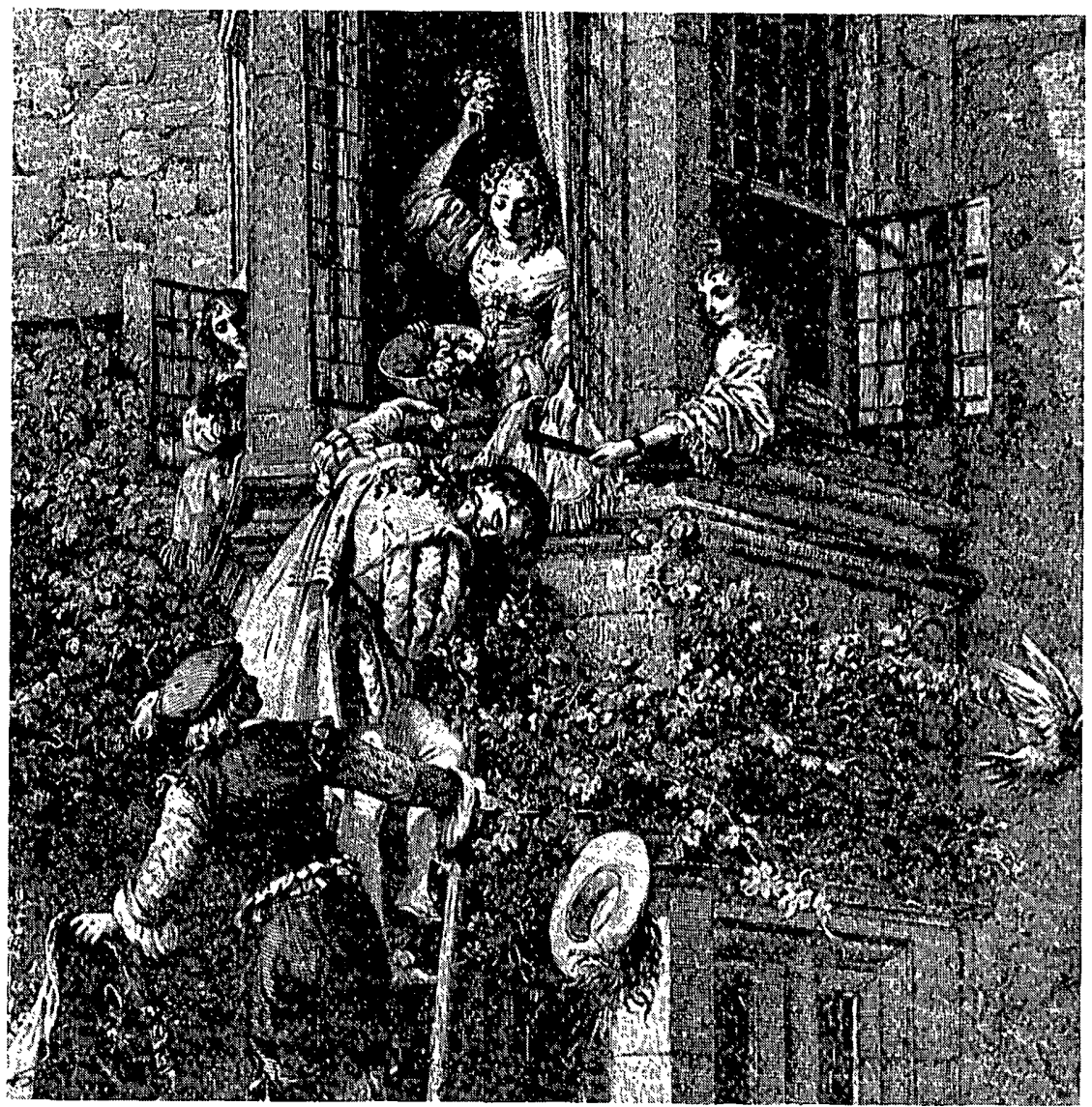

